\title{
CRISE TIREOTÓXICA
}

\author{
THYROID STORM
}

Léa M. Z. Maciel

Docente. Divisão de Endocrinologia. Departamento de Clinica Médica. Faculdade de Medicina de Ribeirão Preto - USP

CorResPondÊnCIA: Av. Bandeirantes, 3900 - CEP 14048 - 900 - Ribeirão Preto - SP. FAX: (16) 6331144 e-mail: Imzmacie@fmrp.usp.br

MACIEL LMZ. Crise tireotóxica. Medicina, Ribeirão Preto, 36: 380-383, abr./dez. 2003.

RESUMO - A crise tireotóxica é uma condição clínica, grave, resultante da exacerbação abrupta do estado hipertireóideo, na qual ocorre descompensação de um ou mais órgãos. Incide com maior freqüência em pacientes com doença de Graves, apesar de poder ocorrer em pacientes com adenoma tóxico ou bócio multinodular tóxico. A apresentação clínica mais comum inclui febre (geralmente $>38,5^{\circ} \mathrm{C}$ ), taquicardia (desproporcional à febre), disfunções gastrointestinais (náuseas, vômitos, diarréia e em casos graves, icterícia), anormalidades neurológicas (variando de confusão a apatia e coma) e hipertensão, seguidas de hipotensão e choque. Apesar de ser rara, o diagnóstico precoce e o tratamento agressivo são necessários para evitar o desfecho fatal, que ocorre em $10 \%$ a $75 \%$ dos pacientes hospitalizados.

UNITERMOS - Crise Tireóidea.

\section{1- INTRODUÇÃO}

A crise tireotóxica (CT) constitui uma exacerbação aguda do estado hipertireóideo e representa o grau máximo de expressão clínica da tireotoxicose. É uma condição rara e fatal, se não diagnosticada rapidamente $^{(1,2,3)}$. Apesar de a CT poder se desenvolver em pacientes hipertireóideos de longa duração, ela se manifesta mais freqüentemente associada a um evento agudo como: período pós-cirurgias, tireoidianas ou não, infecção, trauma, sobrecarga aguda de iodo, uso de drogas anticolinérgicas e adrenérgicas, gravidez, ingestão de hormônio tireoidiano, embolia pulmonar, cetoacidose diabética ou acidente vascular cerebral. Apesar de a literatura ${ }^{(2 / 5)}$ sugerir que a administração de iodo radioativo deva ser considerada como um fator desencadeante, investigações recentes ${ }^{(6,7,8)}$ colocam em dúvida tal afirmação, apontando a suspensão da droga antitireoidiana, previamente à administração do iodo, como sendo o principal fator responsável, pelo desencadeamento da crise.

\section{2- PATOGÊNESE}

Desconhecida, ainda que algumas teorias tenham sido propostas, como as mostradas a seguir:

- Elevação rápida das concentrações dos hormônios tireoidianos, livres ${ }^{(9)}$, decorrentes de alterações nas concentrações de proteínas carreadoras desses hormônios ${ }^{(10)}$ ou da produção de inibidores de ligação do hormônio tireoidiano a essas proteínas, como acontece na Síndrome do T3 baixo ${ }^{(11)}$. Outra possibilidade seria a liberação rápida dos hormônios, associada a manipulação cirúrgica ${ }^{(12)}$.

- Participação do Sistema Nervoso Adrenérgico, apesar das concentrações normais de catecolaminas circulantes, detectadas nos pacientes. Entretanto, a dramática melhora clínica, com agentes que depletam as concentrações de catecolaminas (como a Reserpina) ou com o uso de $\beta$ bloqueadores, sugere sua participação ${ }^{(13)}$. 


\section{3- APRESENTAÇÃO CLÍNICA}

Geralmente, os pacientes apresentam história de hipertireoidismo e mostram as características clínicas da síndrome, exacerbadas e com maior gravidade. Burch \& Wartofsky ${ }^{(14)}$, em revisão de casos publicados, observaram que todos os pacientes apresentavam hipertermia marcante, taquicardia e disfunção do sistema nervoso central. Os autores propuseram os seguintes critérios diagnósticos. (Tabela I)

\section{4- AVALIAÇÃO LABORATORIAL}

Não se pode esquecer que o diagnóstico da Crise Tireotóxica é clínico e que não existe teste laboratorial específico que identifique tal condição. Se o paciente apresentar quadro clínico compatível, não se deve retardar o início do tratamento, aguardando testes laboratoriais. (Tabela II)

\section{5- TRATAMENTO}

Suporte em unidade de terapia intensiva é essencial. Os princípios do tratamento, descritos abaixo são aplicados para os pacientes com hipertireoidismo grave, mesmo que não preencham os critérios diagnósticos para a CT.

\section{Medidas Gerais}

- Alguns pacientes poderão requerer administração substancial de líquidos, enquanto, em outros, a administração deverá ser mais cuidadosa; estão indicados diuréticos em pacientes com ICC.

- Digital - necessidade de doses altas nos pacientes com diagnóstico de ICC.

- Hiperpirexia - deverá ser agressivamente corrigida. A administração de acetoaminofeno é preferível à de aspirina, devido ao aumento das concentrações das frações livres de T3 e T4 que esta droga induz, em decorrência da interferência na ligação às proteínas carreadoras ${ }^{(15)}$.

- Busca ativa por focos infecciosos.

Tratamento Medicamentoso: consiste em administração de múltiplas medicações, cada uma delas com diferentes mecanismos de ação, como as indicadas a seguir.

- Betabloqueadores - proporcionam o controle dos sintomas induzidos pelo aumento do tônus adrenér-

Tabela I: Critérios diagnósticos para Crise Tireotóxica

Disfunção termorregulatória

Temperatura $\left({ }^{\circ} \mathbf{C}\right)$

$37,2-37,7$

$37,8-38,2$

$38,3-38,8$

$38,8-39,4$

$39,5-39,9$

$\geq 40$

Efeito Sistema Nervoso Central

- Ausente

- Leve - agitação

- Moderada - delírio, psicose, letargia extrema

- Grave - convulsões, coma

Disfunção gastrointestinal e hepática

- Ausente

- Moderada: diarréia, náusea/vômito, dor abdominal

- Grave: icterícia não explicada

Pontos

Pontos

5

10

15

20

25

30

Score $\geq 45$ - é diagnóstico de crise tireotóxica

Score 25 a 44 - é compatível com o diagnóstico de crise tireotóxica iminente

Score $<25$ - diagnóstico de crise tireotóxica pouco provável
Disfunção cardiovascular

Pontos

\section{Taquicardia}

99-109

5

110-119

10

120-129

15

$130-139$

20

$\geq 140$

25

Insuficiência Cardíaca Congestiva

- Ausente

0

- Leve

5

- Moderada

10

- Grave (edema pulmonar)

Fibrilação atrial

10

História precipitante

- Negativa

0

- Positiva 
Tabela II: Avaliação laboratorial de pacientes com crise tireotóxica.

Exames

TSH

Supresso (TSH será normal ou elevado, se a etiologia for excesso de secreção de TSH por tumor hipofisário).

T3 e T4 (total ou livre)

Elevados

Eletrólitos

Normais. Cálcio poderá estar elevado pela hemoconcentração ou pelos efeitos dos hormônios tireoidianos sobre o osso

Leucócitos

Leucocitose com desvio à esquerda, mesmo na ausência de infecção

Glicemia

Hiperglicemia leve a moderada, mesmo na ausência de Diabetes Mellitus, provavelmente pelo aumento da glicogenólise e da liberação de insulina mediada por catecolaminas.

Desidrogenase láctica, TGO, bilirrubinas Elevadas como decorrência de disfunção hepática.

Elevado na crise tireotóxica, como em qualquer outra situação estressante, porém, se o resultado for normal, deverá ser interpretado como

Cortisol sérico inapropriadamente baixo, compatível com insuficiência adrenal ou por incapacidade da glândula adrenal em atender à demanda decorrente do acelerado turnover dos glicocorticóides

Eletrocardiograma

Taquicardia, fibrilação atrial

RX de tórax

Poderá evidenciar aumento da área cardíaca e congestão pulmonar devido a ICC ou velamentos associados à infecção pulmonar

Tomografia computadorizada de crânio

Deverá ser realizada para excluir outras condições neurológicas, se o diagnóstico for incerto e nos pacientes com alterações neurológicas, após a estabilização inicial do paciente

gico e é de grande importância no tratamento, pois o controle da frequiência cardíaca levará à melhora da função cardíaca global. Entretanto, deverão ser observadas outras situações nas quais existe contra-indicação para o seu uso, por ex: asma brônquica.

Propranolol: administração endovenosa (EV), $1 \mathrm{mg} / \mathrm{min}$, até que o $\beta$ bloqueio seja alcançado ${ }^{(16)} \mathrm{ou}$, por via oral ou sonda nasogástrica, na dose de 60-80 mg a cada $4 \mathrm{~h}$. Outra alternativa é a utilização de $\beta$ bloqueador de curta duração - esmolol - Dose de ataque 250 - $500 \mathrm{mg} / \mathrm{Kg}$, seguida de infusão de $50-100$ $\mathrm{mcg} / \mathrm{min} .{ }^{(17)}$.

- Tionamidas: administradas com a finalidade de bloquear a síntese de novo dos hormônios tireoidianos, o que ocorre 1 a 2 h após a sua administração. Entretanto, não tem efeito na liberação de hormônios pré-formados pela glândula. Alguns preferem o uso do Propiltiouracil, na CT, devido a sua atuação no bloqueio da conversão periférica de T4 em T3 ${ }^{(18)}$.
Dose: Metimazole: $30 \mathrm{mg}$ a cada $6 \mathrm{~h}$, por via oral ou sonda nasogástrica.

Propiltiouracil: $200 \mathrm{mg}$ a cada $4 \mathrm{~h}$, por via oral ou sonda nasogástrica.

Doses menores poderão ser utilizadas em pacientes que não preencham os critérios para CT, mas que apresentem hipertireoidismo grave.

- Contrastes radiológicos ou soluções iodadas: ácido iopanóico ou outro contraste radiológico. São potentes inibidores da conversão periférica de $\mathrm{T} 4$ em T3 ${ }^{(19)}$. Devem ser administrados pelo menos $1 \mathrm{~h}$ após a administração das tionamidas.

Dose: 0,5 a 1,0 gramas, por via oral, diariamente. Solução de Lugol - 10 gotas, 3 vezes ao dia ou SSKI - 5 gotas a cada $6 \mathrm{~h}$.

- Glicocorticóides - administrados no sentido de reduzir a conversão de T4 a T3 ${ }^{(20)}$.

- Hidrocortisona: $100 \mathrm{mg}$, EV, a cada $8 \mathrm{~h}$, apenas nos casos sugestivos de crise tireotóxica. Nos pa- 
cientes com hipertireoidismo grave, que não corram risco de vida, a hidrocortisona não é indicada.

- Lítio: tem sido utilizado para bloquear a liberação aguda do hormônio tireoidiano, entretanto, a sua toxicidade renal e neurológica tem limitado a sua utilização ${ }^{(17)}$.

- Plasmaferese: tem sido utilizada quando o tratamento tradicional não é bem sucedido ${ }^{(21)}$.

MACIEL LMZ. Thyroid storm. Medicina, Ribeirão Preto, 36: 380-383, apr./dec. 2003.

ABSTRACT - Thyroid storm is a relatively rare but life-threatening exacerbation of the hyperthyroid state in which there is evidence of decompensation in one or more organs. Thyroid storm is most frequently seen in patients with thyrotoxicosis secondary to Graves's disease, although it can occurs in patients with toxic adenoma or toxic multinodular goiter. Common clinical presentation includes fever (temperature usually $>38,5^{\circ} \mathrm{C}$ ), tachycardia (out the proportion of the fever), gastrointestinal dysfunction (including nausea, vomiting, diarrhea and in severe cases, jaundice), neurological abnormalities, varying from confusion to apathy and even coma and hypertension, followed by hypotension and shock. Despite its relative rarity, early diagnosis and vigorous therapy are required to avoid a fatal outcome; mortality rates of hospitalized patients range from $10 \%$ to $75 \%$.

UNITERMS - Thyroid Crisis.

\section{REFERÊNCIAS BIBLIOGRÁFICAS}

1 - INGBAR SH. Management of emergencies. IX. Thyrotoxic storm. N Engl J Med 274: 1252-1254, 1966.

2 - MCDERMOTT MT; KIDD GS; DODSON LE JR \& HOFELDT FD. Radioiodine-induced thyroid storm. Case report and literature review. Am J Med 75: 353-359, 1983.

3 - BURCH HB \& WARTOFSKY L. Life-threating thyrotoxicosis. Thyroid storm. Endocrinol Metab Clin North Am 22:263-277, 1993.

4 - HAYEK A. Thyroid storm following radioiodine for thyroxicosis. J Pediatr 93: 978-980, 1978.

5 - CERLETTY JM \& LISTAW AN. Hyperthyroidism due to functioning metastatic thyroid carcinoma. Precipitation of thyroid storm with therapeutic radioactive iodine. JAMA 242: 269-270, 1979.

6 - ANDRADE VA; GROSS JL \& MAIA AL. Effect of methimazole pretreatment on serum thyroid hormone levels after radioactive treatment in Graves' hyperthyroidism. J Clin Endocrinol Metab 84: 4012-4016, 1999.

7 - BURCH HB; SOLOMON BL; COOPER DS; FERGUSON P; WALPERT N \& HOWARD R. The effect of antithyroid drug pretreatment on acute changes in thyroid hormone levels after (131)I ablation for Graves' disease. J Clin Endocrinol Metab 86: 3016-3021, 2001.

8 - KADMONPM; NOTO RB; BONEY CM; GOODWING \& GRUPPUSO PA. Thyroid storm in a child following radioactive iodine (RAI) therapy: a consequence of RAI versus withdrawal of antithyroid medication. J Clin Endocrinol Metab 86:1865-1867, 2001.

9 - BROOKS MH \& WALDSTEIN SS. Free thyroxine concentrations in thyroid storm. Ann Intern Med 93: 694-697, 1980.

10 - COLEBUNDERS R; BOURDOUX P; BEKAERT J; MAHLER C \& PARIZEL $G$. Determination of free thyroid hormones and their binding proteins in a patient with severe hyperthyroidism (thyroid storm?) and thyroid encephalopathy. J Endocrinol Invest 7: 379-381, 1984.
11 - ROTH RN \& MCAULIFFE MJ. Hyperthyroidism and thyroid storm. Emerg Med Clin North Am 7: 873-883, 1989.

12 - TIETGENS S \& KEINUNG M. Thyroid Storm. Med Clin North Am 79: 169-184, 1995.

13 - DAS G \& KREIGER M. Treatment of thyrotoxic storm with intravenous administration of propranolol. Ann Intern Med 70: 985-988, 1969.

14 - BURCH HB \& WARTOFSKY L. Life-threatening thyrotoxicosis: Thyroid Storm. Endocrinol Metab Clin North Am 22 263-277, 1993.

15 - CHIOVATO L; BARBESINO G \& PINCHERA A. Graves' Disease In: DEGROOT L \& JAMESON JL, eds. Endocrinology. $4^{\text {th }}$ ed, W.B. Saunders, Philadelphia, p.1422-1443, 2001.

16 - LARSEN PR. Salicylate-induced increases in free triiodothyronine in human serum. Evidence of inhibition of triiodothyronine binding to thyroxine-binding globulin and thyroxine-binding prealbumin. J Clin Invest 51: 1125-1134, 1972.

17 - BRUNETTE DD \& ROTHONG C. Emergency department management of thyrotoxic crisis with esmolol. Am J Emerg Med 9: 232-234, 1991.

18 - HENNEMAN G. Graves' disease: Complications. thyroid storm. In: The thyroid and its diseases. Http:// www.thyroidmanager.org/Chapter 12.

19 - ROTI E; GARDINI E; MINELLI R; SALVI M; ROBUSCHI G \& BRAVERMAN LE. Methimazole and serum thyroid hormone concentrations in hyperthyroid patients: effects of single and multiple daily doses. Ann Intern Med 111: 181-182, 1989.

20 - MAZZAFERRI EL \& SKILLMAN TG. Thyroid storm. A review of 22 episodes with special emphasis on the use of guanethidine. Arch Intern Med 124: 684-690, 1969.

21 - CANDRINA R; DI STEFANO O; SPANDRIO S \& GIUSTINA G Treatment of thyrotoxic storm by charcoal plasmaperfusion. J Endocrinol Invest 12: 133-134, 1989. 